\title{
Overexpression of heart-type fatty acid binding protein enhances fatty acid-induced podocyte injury
}

\author{
QING GAO $^{1}$, ALHOSSAIN SARKAR ${ }^{1}$, YIZHI $\mathrm{CHEN}^{2,3}$, BO XU $^{1}$, \\ XIAOJUAN ZHU ${ }^{1}$, YANG YUAN ${ }^{1}$ and TIANJUN GUAN ${ }^{1}$ \\ ${ }^{1}$ Department of Nephrology, Zhongshan Hospital, Xiamen University, Xiamen, Fujian 361004; \\ ${ }^{2}$ Department of Nephrology, Hainan Branch of Chinese PLA General Hospital, Sanya, Hainan 572013; \\ ${ }^{3}$ Department of Nephrology, Chinese PLA General Hospital, Chinese PLA Institute of Nephrology, Beijing 100086, P.R. China
}

Received April 6, 2017; Accepted August 17, 2017

DOI: $10.3892 /$ etm.2017.5643

\begin{abstract}
Deregulated lipid metabolism is a characteristic of metabolic diseases including type 2 diabetes and obesity, and likely contributes to podocyte injury and end-stage kidney disease. Heart-type fatty acid binding protein (H-FABP) was reported to be associated with lipid metabolism. The present study investigated whether H-FABP contributes to podocyte homeostasis. Podocytes were transfected by lentiviral vector to construct a cell line which stably overexpressed H-FABP. Small interfering RNA capable of effectively silencing H-FABP was introduced into podocytes to construct a cell line with H-FABP knockdown. Certain groups were treated with palmitic acid (PA) and the fat metabolism, as well as inflammatory and oxidative stress markers were measured. PA accelerated lipid metabolism derangement, inflammatory reaction and oxidative stress in podocytes. Overexpression of H-FABP enhanced the PA-induced disequilibrium in podocytes. The mRNA and protein expression levels of acyl-coenzyme A oxidase 3 and monocyte chemotactic protein 1 , and the protein expression levels of 8-hydroxy-2'-deoxyguanosine and 4-hydroxynonenal were upregulated in the H-FABP overexpression group, while the mRNA and protein expression of peroxisome proliferator activated receptor $\alpha$ was downregulated. Knockdown of H-FABP inhibited the PA-induced injury and lipid metabolism derangement, as well as the inflammatory reaction and oxidative stress in podocytes. These results indicated that overexpression of H-FABP enhances fatty acid-induced podocyte injury, while H-FABP inhibition may represent a potential therapeutic strategy for the prevention of lipid metabolism-associated podocyte injury.
\end{abstract}

Correspondence to: Dr Tianjun Guan, Department of Nephrology, Zhongshan Hospital, Xiamen University, 210-219 South Hubin Road, Xiamen, Fujian 361004, P.R. China

E-mail: guantianjun@aliyun.com

Key words: heart-type fatty acid binding protein, fat metabolism, inflammation, oxidative stress, podocytes

\section{Introduction}

Podocyte injury critically contributes to the pathogenesis of proteinuric kidney diseases, including diabetic nephropathy and obesity-associated glomerulopathy (ORG). Deregulation of the lipid metabolism with a disturbed free fatty acid (FFA) metabolism is a characteristic of metabolic disorders including type 2 diabetes and obesity and likely contributes to end-stage kidney disease irrespective of the underlying kidney disease (1). From a lipotoxic-podocyte-centric viewpoint, podocin and nephrin must be spatially inserted within the cholesterol-rich segments (lipid rafts) of the podocyte slit diaphragm to maintain proper podocin-nephrin and podocin-transient receptor potential cation channel 6 (TRPC-6) interactions, as well as podocyte functions. Dysregulated fatty acid oxidation affects podocin palmitoylation and thus proper membrane insertion; fatty acid overload may cause altered lipid composition of the lipid rafts and therefore interferes with network signaling of the podocin-nephrin-TRPC- 6 actin cytoskeleton, triggering a cascade of well-described downstream events that have pathologic effects. Lipids are essential for maintaining the function of renal cells, while lipid metabolism derangement induces an inflammatory reaction, oxidative stress, fibrous degeneration and necrosis on renal tissue (2-4).

Fatty acid binding proteins (FABPs) have a role in lipid metabolism. Nonenzymatic cytosolic FABPs are abundantly expressed in numerous animal tissues with high rates of fatty acid metabolism. FABP has 10 isoforms that are distinctly distributed in various tissues in mammals and that have distinct mechanisms in different organs and cells (5). Among them, heart-type (H)-FABP, also referred to as FABP3, is most predominantly expressed in the heart and less in the skeletal muscle, kidney, stomach and testis (5). Kimura et al (6) first reported that H-FABP is present in human glomeruli and is localized largely along the capillary wall. To date, most studies on H-FABP have focused on the heart, while the role of H-FABP in the kidney has remained largely elusive.

A previous study by our group reported that H-FABP expression was significantly higher on podocytes of ORG patients than on those of normal controls. In addition, increased levels of H-FABP were correlated with the progression of proteinuria in patients with ORG (7). However, the 
function of H-FABP in podocytes has remained to be fully elucidated. Accordingly, the present study investigated the effect of H-FABP on lipid metabolism, inflammatory reaction and oxidative stress in podocytes.

\section{Materials and methods}

Cell culture. Conditionally immortalized murine podocytes [heat-sensitive mouse podocytes (HSMPs)] were provided Professor Stuart Shankland (Division of Nephrology, University of Washington, Seattle, WA, USA) (8). The cells were grown at $33^{\circ} \mathrm{C}$ in RPMI 1640 medium (Hyclone; GE Healthcare, Little Chalfont, UK) containing 10\% heat-inactivated fetal calf serum (Gibco; Thermo Fisher Scientific, Inc., Waltham, MA, USA), $100 \mathrm{U} / \mathrm{ml}$ penicillin $\mathrm{G}, 100 \mu \mathrm{g} / \mathrm{ml}$ streptomycin and $10 \mathrm{U} / \mathrm{ml}$ recombinant murine interferon- $\gamma$ (IFN- $\gamma$; Sigma-Aldrich; Merck KGaA, Darmstadt, Germany) in the presence of $5 \% \mathrm{CO}_{2}$. Proliferating cells were cultured at $37^{\circ} \mathrm{C}$ in IFN- $\gamma$-free medium for 14 days to induce differentiation.

Establishment of cell line overexpressing H-FABP. Lentivirus overexpressing H-FABP and green fluorescent protein (GFP) was obtained from Shanghai GenePharma (Shanghai, China) with a multiplicity of infection (MOI) of $1 \times 10^{8}$. Differentiated HSMPs were seeded in 6-well plates at $1 \times 10^{5}$ cells per well. After incubation overnight, the lentivirus (MOI, $1 \times 10^{7}$ ) or control vector, which is vacuous vector, was applied to each well using Fugene 6 Xtreme transfection reagent (Roche Diagnostics, Basel, Switzerland). Transfected cells were incubated at $37^{\circ} \mathrm{C}$ for $48 \mathrm{~h}$ and the media was replaced. At 2 weeks post-transfection, successfully transfected cells were selected with puromycin ( $1 \mu \mathrm{g} / \mathrm{ml}$ final concentration). The transfection efficiency was observed by detecting GFP by fluorescence microscopy.

H-FABP small interfering (si)RNA transfection. Prior to transfection, differentiated HSMPs were seeded in 6-well plates at $1 \times 10^{5}$ cells per well. Subsequently, $92 \mu \mathrm{H}_{2} \mathrm{O}+5 \mu \mathrm{l}$ siRNA or negative siRNA (Shanghai GenePharma) $+3 \mu$ l HiPerfect transfection reagent (Shanghai GenePharma) was applied to each well, followed by incubation at $37^{\circ} \mathrm{C}$ for $24 \mathrm{~h}$. In the present study, two different siRNAs for H-FABP were used and the nucleotide fragments had the following sequences: Fabp3-Mus-298, 5'-GCAGGAGACAACACUAACUTT-3'; and Fabp3-Mus-369, 5'-GTGAGCACTCGGACTTAT-3'. RT-qPCR and western blot analysis were used to evaluate the expression levels of H-FABP. Which one had a higher inhibitory efficacy was selected for use in the subsequent experiments.

Cell grouping. Prior to the experiments, differentiated HSMPs were seeded in 6 -well plates at $2 \times 10^{5}$ cell per well. On the following day, siRNA transfection and/or palmitic acid (PA; final concentration, $30 \mu \mathrm{mol} / \mathrm{l}$ ) were applied to each well. In addition, mismatched clones were transfected into differentiated HSMPs cells as a negative control group. Untransfected cells were used as a normal control. Successfully transfected lentivirus overexpressing H-FABP cells were used as stably overexpressed H-FABP cells. The cells were then divided into ten groups: i), Normal control group; ii) normal control+PA group; iii) si-negative control group; iv) si-H-FABP group; v) si-negative control+PA group; vi) si-H-FABP+PA group; vii) negative control group; viii) H-FABP overexpression group; ix) negative control+PA group; $\mathrm{x}$ ) H-FABP overexpression+PA group. All these cells were treated for $48 \mathrm{~h}$, and RNA and protein were then collected.

$R T$-qPCR analysis. Total RNA was extracted from cultured cells using TRIzol ${ }^{\circledR}$ reagent (Invitrogen; Thermo Fisher Scientific, Inc.). Single-stranded complementary (c)DNA was generated from DNaseI-treated RNA samples using Moloney murine leukemiavirus reverse transcriptase and oligo $(\mathrm{dT})_{12-18}$ (Toyobo Life Science, Osaka, Japan). A total of $1 \mu \mathrm{l}$ cDNA samples were used for conventional PCR amplifications. For qPCR analysis, PCR reactions were performed in a real-time PCR system (StepOne, Applied Biosystems; Thermo Fisher Scientific, Inc.) and the expression levels of target genes relative to $\beta$-actin were determined using a SYBR Green-based comparative cycle threshold CT method (9). The following thermocycling conditions were used: $95^{\circ} \mathrm{C}$ for $10 \mathrm{~min}, 95^{\circ} \mathrm{C}$ for $5 \mathrm{sec}$ and $60^{\circ} \mathrm{C}$ for $1 \mathrm{~min}$ for 40 cycles, followed by $95^{\circ} \mathrm{C}$ for $15 \mathrm{sec}, 60^{\circ} \mathrm{C}$ for $1 \mathrm{~min}$ and $95^{\circ} \mathrm{C}$ for $15 \mathrm{sec}$ for melt curve analysis, followed by an additional incubation at $72^{\circ} \mathrm{C}$ for $1 \mathrm{~min}$ for elongation in the final cycle. The primer sequences are listed in Table I.

Western blot analysis. The cells were rinsed with ice-cold PBS and lysed in radioimmunoprecipitation assay buffer (150 mM sodium chloride, $1.0 \%$ Triton $\mathrm{X}-100,0.5 \%$ sodium deoxycholate, $0.1 \%$ sodium dodecyl sulfate and $50 \mathrm{mM}$ Tris, $\mathrm{pH}$ 8.0) mixed with protease inhibitors (cat. no. P8340; Sigma-Aldrich; Merck KGaA) for 30 min on ice. Samples were collected and centrifuged at $13,800 \mathrm{x}$ g for $10 \mathrm{~min}$ at $4^{\circ} \mathrm{C}$. Supernatant protein was boiled in loading buffer at $100^{\circ} \mathrm{C}$ for $10 \mathrm{~min}$. Protein concentration was determined using the BCA method. The protein samples (20 $\mu \mathrm{g}$ per lane) were subjected to $15 \%$ SDS-PAGE and transferred onto nitrocellulose membranes (GE Healthcare). After blocking with milk, membranes were incubated with primary antibodies, including peroxisome proliferator activated receptor $\alpha(\operatorname{PPAR} \alpha)$ rabbit anti-mouse polyclonal antibody (1:1,000 dilution; ab8934; Abcam, Cambridge, MA, USA), acyl-coenzyme A oxidase 3 (ACOX3) rabbit anti-mouse polyclonal antibody $(1: 1,000$ dilution; PA5-22373; Pierce,Thermo Fisher Scientific, Inc.), monocyte chemotactic protein 1 (MCP-1) rabbit anti-mouse polyclonal antibody, (1:1,000 dilution; ab7202; Abcam), 4-hydroxynonenal (4-HNE) rabbit anti-mouse polyclonal antibody (1:1,000 dilution; ab46545; Abcam), 8-hydroxy-2'deoxyguanosine $(8-\mathrm{OHdG})$ rabbit anti-mouse polyclonal antibody (1:1,000 dilution; 251640; Abbiotec LLC, San Diego, CA, USA) and $\beta$-actin mouse monoclonal antibody as a control (1:1,000 dilution; sc-130300; Santa Cruz Biotechnology, Inc., Dallas, TX, USA) overnight at $4^{\circ} \mathrm{C}$. HRP-conjugated purified goat anti-rabbit immunoglobulin $\mathrm{G}$ (1:5,000 dilution; $\mathrm{P} / \mathrm{N}$ 926-80011; LI-COR Biosciences, Lincoln, NE, USA) was used as a secondary antibody for $1 \mathrm{~h}$ at room temperature. The bands were visualized with a ChemiDOC XRS imaging system (Bio-Rad Laboratories, Inc., Hercules, CA, USA). Densitometry was performed on all gels and each signal was 
Table I. Primer sequences for polymerase chain reaction.

\begin{tabular}{|c|c|c|c|}
\hline Gene & Direction & Primer sequence (5'-3') & $\begin{array}{l}\text { Product } \\
\text { length }(\mathrm{bp})\end{array}$ \\
\hline \multirow[t]{2}{*}{ H-FABP } & Forward & $\begin{array}{l}\text { GACCAAGCCTACTAC } \\
\text { CATCATC }\end{array}$ & 117 \\
\hline & Reverse & $\begin{array}{l}\text { GTCACCTCGTCGAAC } \\
\text { TCTATTC }\end{array}$ & \\
\hline \multirow[t]{2}{*}{ MCP-1 } & Forward & $\begin{array}{l}\text { CCCACTCACCTGCTG } \\
\text { СТАСТ }\end{array}$ & 164 \\
\hline & Reverse & $\begin{array}{l}\text { TCTGGACCCATTCCT } \\
\text { TCTTG }\end{array}$ & \\
\hline \multirow[t]{2}{*}{$\operatorname{PPAR} \alpha$} & Forward & $\begin{array}{l}\text { CGGTGTGTATGAAGC } \\
\text { СATCT }\end{array}$ & 101 \\
\hline & Reverse & $\begin{array}{l}\text { TAAGGAACTCGCGTG } \\
\text { TGATAAA }\end{array}$ & \\
\hline \multirow[t]{2}{*}{ ACOX3 } & Forward & $\begin{array}{l}\text { ACCCACGGATAAGGA } \\
\text { AGAGA }\end{array}$ & 99 \\
\hline & Reverse & $\begin{array}{l}\text { GAGAAGTGGTCCAAG } \\
\text { GCATAG }\end{array}$ & \\
\hline
\end{tabular}

H-FABP, heart-type fatty acid binding protein; ACOX3, acyl-coenzyme A oxidase 3; MCP-1, monocyte chemotactic protein 1; PPAR $\alpha$, peroxisome proliferator activated receptor $\alpha$.

normalized to the corresponding $\beta$-actin signal using Quantity One Image Analysis software (version number 4.6.6, Bio-Rad Laboratories, Inc.).

Statistical analysis. All values were expressed as the mean \pm standard deviation and analyzed with SPSS 17.0 (SPSS, Inc., Chicago, IL, USA). Differences in mean values were assessed using Student's t-test or one-way analysis of variance. Multiple comparison between the groups was performed using the Student-Newman-Keuls method. $\mathrm{P}<0.05$ was considered to indicate a statistically significant difference.

\section{Results}

Overexpression and knockdown of H-FABP in HSMPs. To confirm the establishment of cells stably transfected with lentiviral vectors, the expression of GFP in the HSMPs in the negative control and H-FABP groups was observed under an inverted fluorescence microscope (Fig. 1). The lentiviral vectors expressed GFP and a similar fluorescence intensity was observed for the two groups, indicating a similar efficacy of lentiviral vector transfection.

Two different siRNAs for H-FABP were used and the nucleotide fragments had the following sequences: Fabp3-Mus-298, 5'-GCAGGAGACAACACUAACUTT-3'; and Fabp3-Mus-369, 5'-GTGAGCACTCGGACTTAT-3'. These small fragments were transfected into HSMPs to inhibit the expression of H-FABP, and the levels of H-FABP were detected by RT-qPCR after $24 \mathrm{~h}$ (Fig. 2). The siRNA causing the lowest expression of H-FABP was selected for the subsequent studies. The results indicated that each of the two fragments successfully inhibited the expression of H-FABP in HSMPs; however, Fabp3-Mus-298 had a higher inhibitory efficacy and was therefore selected for use in the subsequent experiments.

In order to further confirm the differential expression levels of H-FABP in the H-FABP overexpression group and the si-H-FABP group, the mRNA and protein levels of H-FABP in these groups were detected (Fig. 3). The results indicated that after transfection with H-FABP overexpression vector, the HSMPs exhibited stable overexpression of H-FABP. After transfection with H-FABP siRNA, the expression of H-FABP in HSMPs was inhibited.

si-H-FABP counteracts PA-induced lipid metabolism disorders in podocytes. To examine the lipid metabolism in podocytes with different expression levels of H-FABP, the mRNA and protein levels of PPAR $\alpha$ and ACOX3 were detected (Fig. 4). The results indicated that after lentiviral vector-mediated overexpression of H-FABP in HSMPs, the mRNA and protein levels of PPAR $\alpha$ were reduced $(\mathrm{P}<0.05)$, but the mRNA and protein levels of ACOX3 were increased $(\mathrm{P}<0.05)$. After transfection of H-FABP siRNA into HSMPs, compared with the normal control group, the mRNA and protein levels of PPAR $\alpha$ were increased $(\mathrm{P}<0.05)$, but the mRNA and protein levels of ACOX3 were reduced $(\mathrm{P}<0.05)$. The mRNA and protein levels of PPAR $\alpha$ were reduced in normal podocytes after PA treatment $(\mathrm{P}<0.05)$, while the mRNA and protein levels of ACOX3 were increased $(\mathrm{P}<0.05)$. Similar results were observed regarding H-FABP overexpressed podocytes after PA treatment $(\mathrm{P}<0.05)$. However, the protein levels of PPAR $\alpha$ were not significantly different in si-H-FABP podocytes after PA treatment $(\mathrm{P}>0.05)$. The mRNA and protein levels of PPAR $\alpha$ in the H-FABP overexpression group were further reduced after PA treatment $(\mathrm{P}<0.05)$, while the mRNA and protein levels of ACOX3 were further increased $(\mathrm{P}<0.05)$. After PA treatment, the mRNA and protein levels of PPAR $\alpha$ in si-H-FABP podocytes were higher than those in normal control podocytes $(\mathrm{P}<0.05)$, while the mRNA and protein levels of ACOX3 were lower than those in the control group $(\mathrm{P}<0.05)$.

si-H-FABP reduces the PA-induced inflammatory reaction in podocytes. To assess the inflammatory reaction, the mRNA and protein levels of MCP-1 in different groups were examined (Fig. 5). The HSMPs in the normal control group exhibited only slight expression of MCP-1. After PA treatment, the mRNA and protein levels of MCP-1 were increased $(\mathrm{P}<0.05)$. After $\mathrm{PA}$ treatment, the protein level of MCP-1 was also increased in $\mathrm{H}-\mathrm{FABP}$ overexpressed podocytes $(\mathrm{P}<0.05)$. However, in si-H-FABP podocytes, the mRNA and protein levels of MCP-1 did not increase after PA treatment. Furthermore, the mRNA and protein levels of MCP-1 were increased after lentiviral vector-mediated overexpression of $\mathrm{H}-\mathrm{FABP}(\mathrm{P}<0.05)$, while they were reduced after transfection with H-FABP siRNA. In the presence of PA, the mRNA and protein levels of MCP-1 in the H-FABP-overexpressing podocytes were higher than those in normal or control-transfected podocytes $(\mathrm{P}<0.05)$. In H-FABP siRNA-transfected podocytes, the mRNA and protein expression of MCP-1 in the presence of PA was lower than that in normal or control-transfected podocytes $(\mathrm{P}<0.05)$. Regardless of the presence of PA, the mRNA and protein 

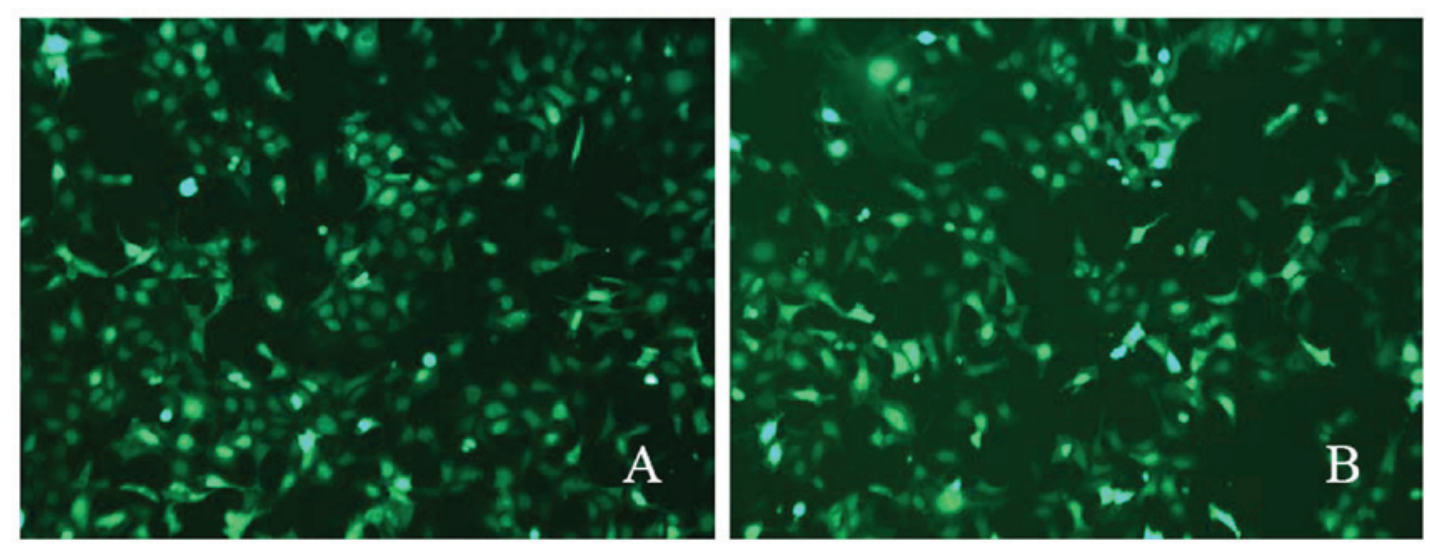

Figure 1. Efficacy of lentiviral expression vector transfection of heat-sensitive mouse podocytes (magnification, x200). (A) Negative control group; (B) heart-type fatty acid binding protein group.

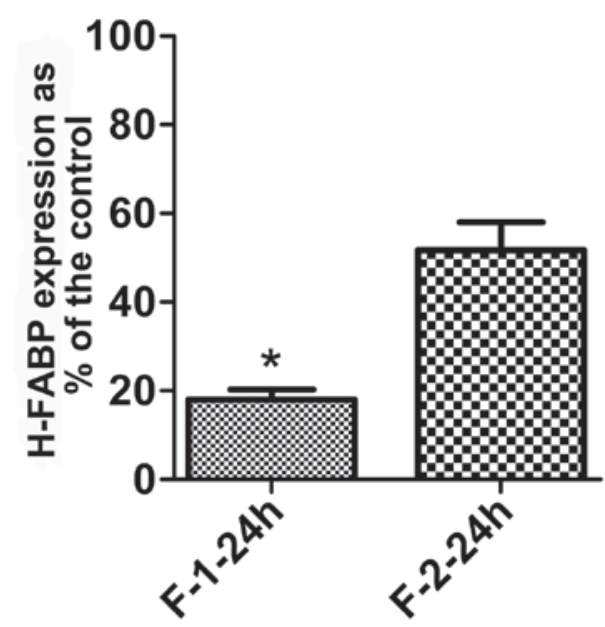

Figure 2. Relative H-FABP mRNA expression levels in HSMPs after transfection of two types of H-FABP siRNA into HSMPs. F-1-24h had a higher inhibitory efficacy. ${ }^{*}<0.05$ vs. F-2-24h group. Groups: F-1-24h, Fabp3-Mus-298 transfected into HSMPs for 24 h; F-2-24h, Fabp3-Mus-369 transfected into HSMPs for $24 \mathrm{~h}$. H-FABP, heart-type fatty acid binding protein; HSMPs, heat-sensitive mouse podocytes.

expression of MCP-1 in podocytes with lentiviral vector-mediated H-FABP overexpression was significantly higher than that in H-FABP siRNA-transfected podocytes $(\mathrm{P}<0.01)$.

si-H-FABP reduces PA-induced oxidative stress in podocytes. Oxidative stress was also examined in the present study. Two indices, 8-OHdG and 4-HNE, were selected to assess oxidative stress (Fig. 6). The results indicated that HSMPs from the normal control group only exhibited slight expression of 8-OHdG and 4-HNE. After PA treatment, the protein levels of $8-\mathrm{OHdG}$ and $4-\mathrm{HNE}$ were increased $(\mathrm{P}<0.05)$. After PA treatment, the protein levels of $8-\mathrm{OHdG}$ and $4-\mathrm{HNE}$ were also increased in H-FABP overexpression podocytes $(\mathrm{P}<0.05)$; however, no significant difference was indicated in si-H-FABP podocytes after PA treatment $(\mathrm{P}>0.05)$. The protein levels of 8-OHdG and 4-HNE were also increased after transfection with H-FABP overexpression vectors $(\mathrm{P}<0.05)$, while they were reduced after transfection with $\mathrm{H}-\mathrm{FABP}$ siRNA. In the presence of PA, the protein expression of 8-OHdG and 4-HNE in the overexpression vector-transfected podocytes was higher

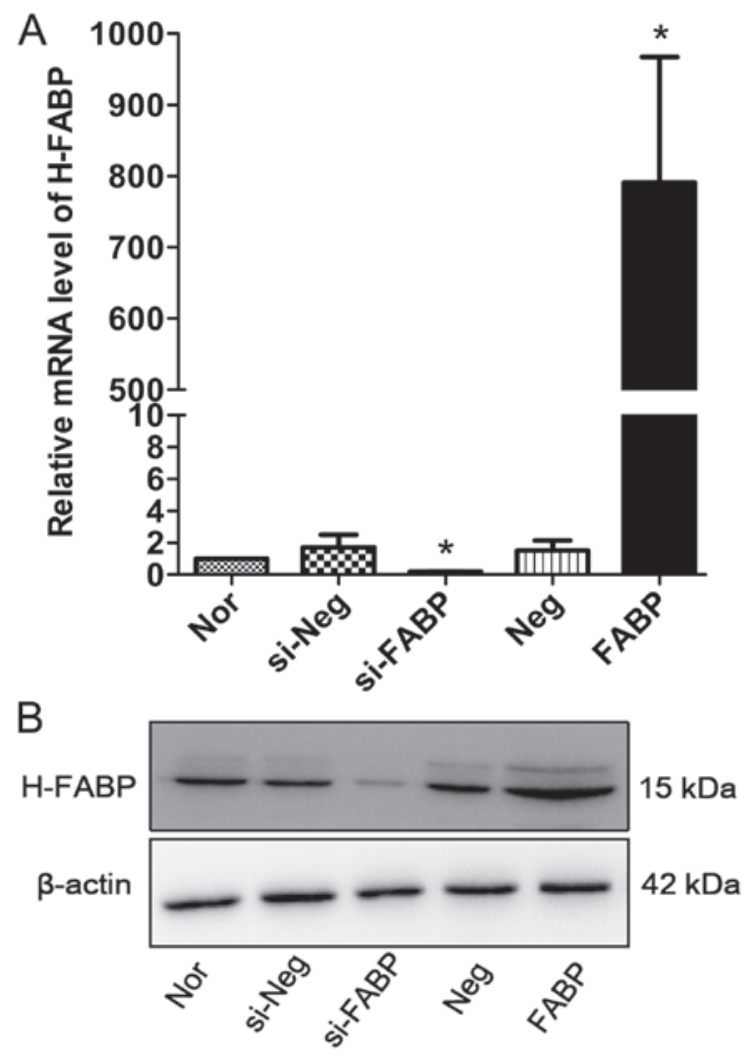

Figure 3. (A) mRNA and (B) protein expression levels of H-FABP in in heat-sensitive mouse podocytes in different groups. ${ }^{*} \mathrm{P}<0.01$ vs. normal control group. Three independent experiments were performed per group. Groups: Nor, normal control group; si-Neg, negative siRNA control group; si-FABP, H-FABP siRNA group; Neg, negative control group; FABP, H-FABP overexpression group. H-FABP, heart-type fatty acid binding protein; siRNA, small interfering RNA.

than that in normal podocytes $(\mathrm{P}<0.05)$, while it was lower than that in normal podocytes in the H-FABP siRNA-transfected podocytes $(\mathrm{P}<0.05)$. Regardless of the presence of PA, the protein expression of $8-\mathrm{OHdG}$ and $4-\mathrm{HNE}$ in the lentiviral H-FABP overexpression vector-transfected podocytes was significantly higher than that in H-FABP siRNA-transfected podocytes $(\mathrm{P}<0.01)$.

The principalfinding of the presentstudy was that overexpression of H-FABP enhanced fatty acid-induced podocyte injury 

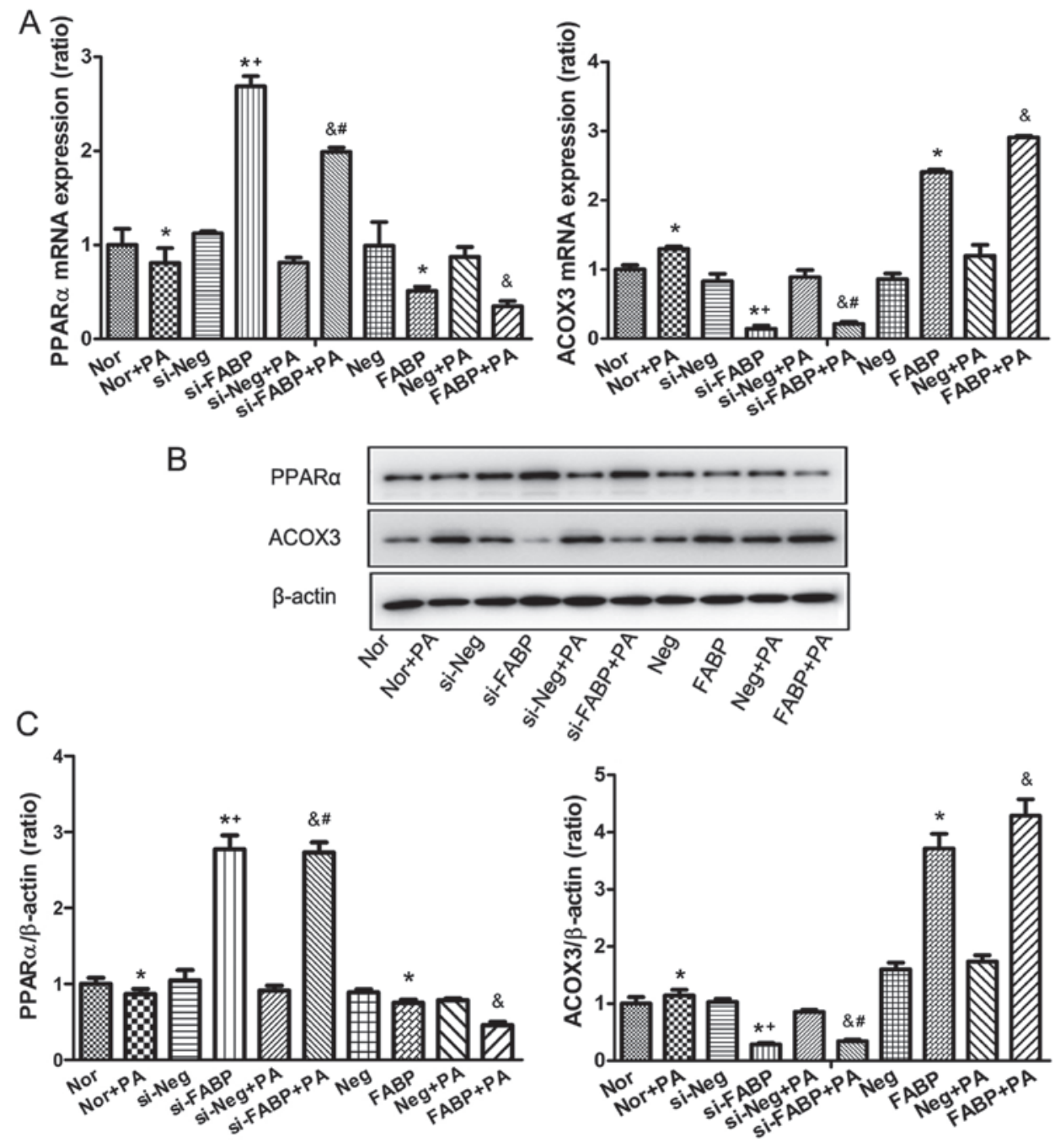

Figure 4. mRNA and protein expression levels of PPAR $\alpha$ and ACOX3. (A) mRNA expression levels of PPAR $\alpha$ and ACOX3 in different groups determined by reverse-transcription quantitative polymerase chain reaction analysis ( $\mathrm{n}=5$ in each group). (B) Representative western blot indicating the protein expression levels of PPAR $\alpha$ and ACOX3 in different groups. (C) Protein levels of PPAR $\alpha$ and ACOX3 in different groups determined by semiquantitative evaluation (n=7 in each group). ${ }^{*} \mathrm{P}<0.05$ vs. normal control group; ${ }^{\&} \mathrm{P}<0.05$ vs. normal control+PA group; ${ }^{+} \mathrm{P}<0.01$ vs. H-FABP overexpression+PA group; ${ }^{*} \mathrm{P}<0.01$ vs. $\mathrm{H}-\mathrm{FABP}$ overexpression+PA group. Groups: Nor, normal control group; Nor+PA, normal control+PA group; si-Neg, negative siRNA control group; si-FABP, H-FABP siRNA group; si-Neg+PA, negative siRNA control+PA group; FABP+PA, H-FABP siRNA+PA group; Neg, negative control group; FABP, H-FABP overexpression group; Neg+PA, negative control+PA group; FABP+PA, H-FABP overexpression +PA group. H-FABP, heart-type fatty acid binding protein; siRNA, small interfering RNA; ACOX3, acyl-coenzyme A oxidase 3; PPAR $\alpha$, peroxisome proliferator activated receptor $\alpha$.

via lipid metabolism derangement, inflammation and oxidative stress. Inhibition of H-FABP expression may counteract fatty acid-induced lipid metabolism derangement, and reduce inflammation and oxidative stress.

\section{Discussion}

Podocytes are specialized epithelial cells of the kidney blood filtration barrier. Several lines of evidence support a dominant role for the impairment of podocytes in glomerular lesions. Variant factors attack podocytes directly or indirectly, including lipid metabolism, infection, immune system derangement, as well as inflammatory and oxidative stress. From a lipotoxic-podocyte-centric viewpoint, lipids are essential for the maintenance of the function of podocytes, while lipid metabolism derangement induces inflammatory reaction, oxidative stress, fibrous degeneration and necrosis of renal tissue (2-4).
The FABP family comprises intracellular proteins of 14-15 kDa in size, which were discovered beginning in the 1970s $(10,11)$. They are a widely expressed and are recognized as a superfamily of small cytosolic proteins that specifically and reversibly bind long-chain fatty acids (LCFAs), and function as the principal vehicles for cytosolic LCFA transport. To date, at least 10 genes encoding FABPs have been identified in the human genome (FABP1-9 and FABP12). FABPs have been indicated to have a wide range of homology (between 20 and $70 \%$ in amino acid sequences) but have similar tertiary structures (12). FABPs all contain a 10-stranded antiparallel $\beta$-barrel structure. The ligand-binding pocket is located inside the $\beta$-barrel and is framed on one side by an $\mathrm{N}$-terminal helix-turn-helix motif that is thought to act as the major portal for long-chain fatty acid entry and exit (13). The expression of different FABPs is distributed through various tissues in a highly specific manner with different levels and functions. The names for the proteins refer to the tissue in which they 

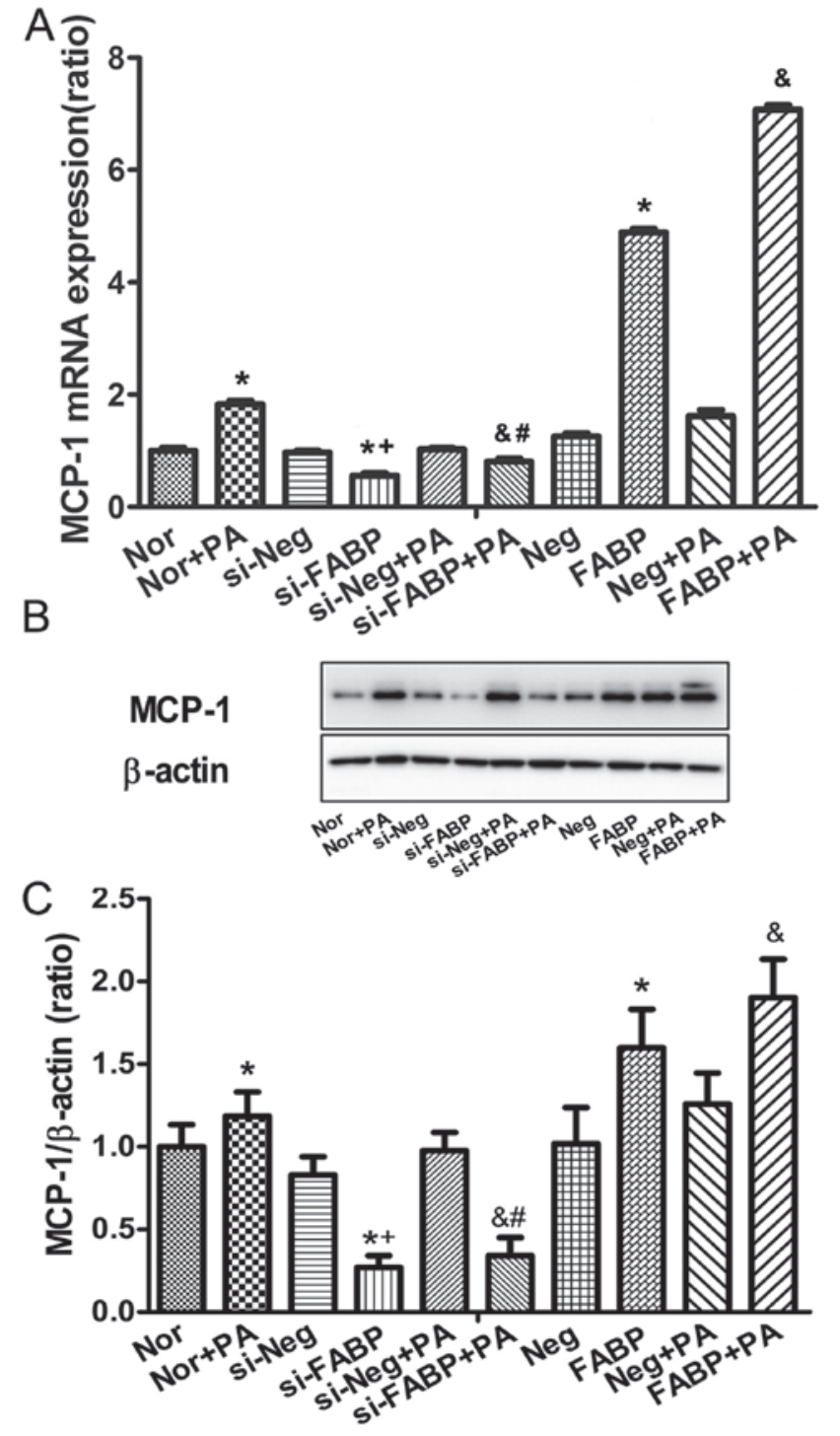

Figure 5. mRNA and protein expression levels of MCP-1. (A) mRNA expression levels of MCP-1 in different groups determined by reverse-transcription quantitative polymerase chain reaction analysis ( $\mathrm{n}=5$ in each group). (B) Representative western blot indicating the protein expression levels of MCP-1 in different groups. (C) Protein levels of MCP-1in different groups determined by semiquantitative evaluation ( $\mathrm{n}=7$ in each group). ${ }^{*} \mathrm{P}<0.05$ vs. normal control group; ${ }^{\circledR} \mathrm{P}<0.05$ vs. normal control+PA group; ${ }^{+} \mathrm{P}<0.01$ vs. H-FABP overexpression; ${ }^{*} \mathrm{P}<0.01$ vs. H-FABP overexpression+PA group. Groups: Nor, normal control group; Nor+PA, normal control+PA group; si-Neg, negative siRNA control group; si-FABP, H-FABP siRNA group; si-Neg+PA, negative siRNA control+PA group; FABP+PA, H-FABP siRNA+PA group; Neg, negative control group; FABP, H-FABP overexpression group; Neg+PA, negative control+PA group; $\mathrm{FABP}+\mathrm{PA}, \mathrm{H}-\mathrm{FABP}$ overexpression +PA group. $\mathrm{H}-\mathrm{FABP}$, heart-type fatty acid binding protein; siRNA, small interfering RNA; MCP-1, monocyte chemotactic protein 1.

were initially identified, but as certain FABPs are expressed in multiple tissues, a numeric nomenclature is also in use (14).

To date, four FABPs have been reported to be mainly associated with the kidney. These include liver-type (L)-FABP, also referred to as FABP1, adipocyte (A)-FABP (also known as FABP4), epidermal (E)-FABP (also called FABP5) and H-FABP. Kamijo-Ikemori et al (15) reported that L-FABP is expressed in human proximal tubules. Guan et al (16) first reported on the presence of A-FABP in renal glomerular cells and renal medullary microvascular endothelial cells.
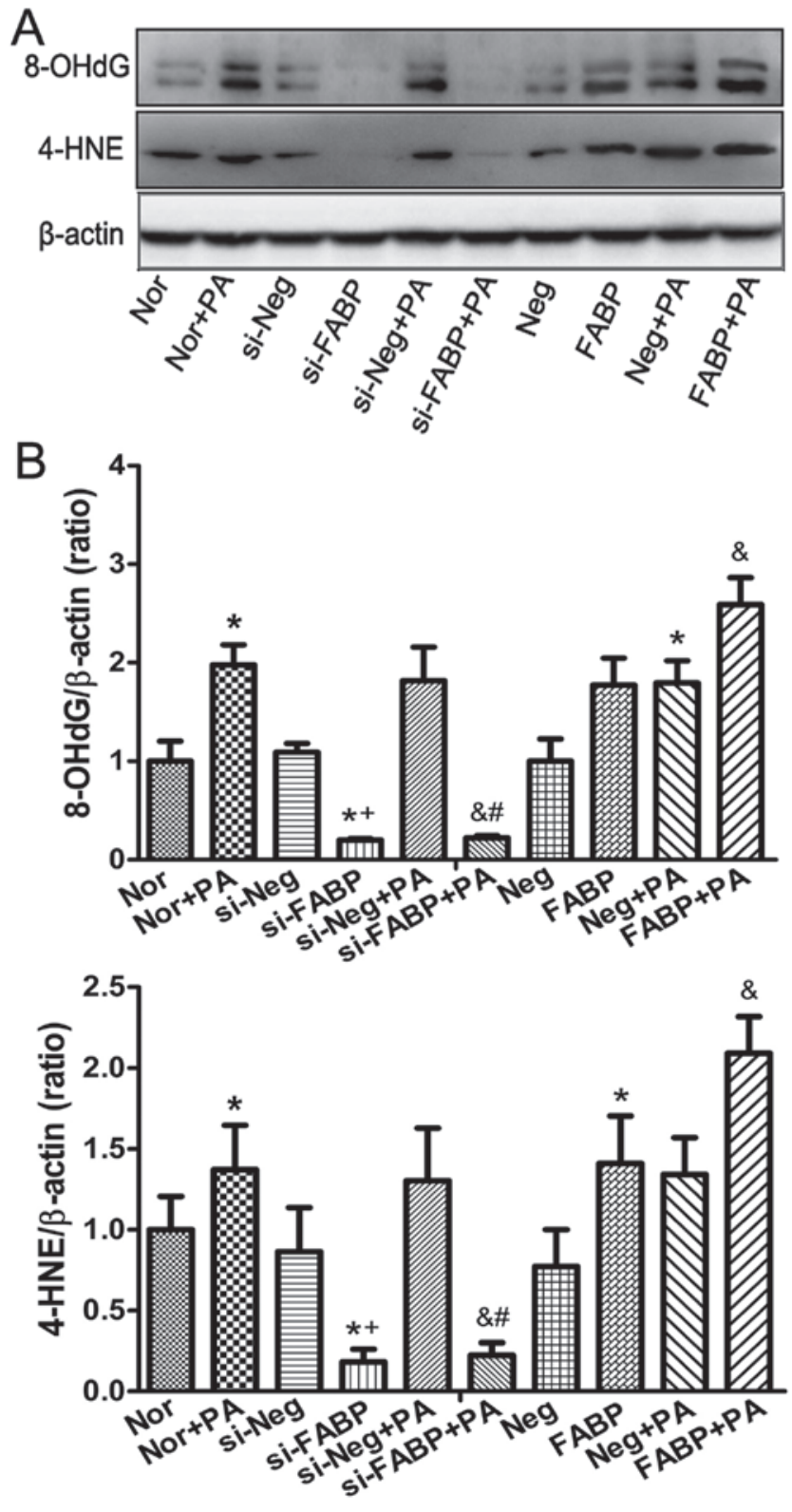

Figure 6. Protein expression levels of 8-OHdG and 4-HNE. (A) Representative western blot indicating the protein expression levels of $8-\mathrm{OHdG}$ and 4-HNE in different groups. (B) Protein levels of $8-\mathrm{OHdG}$ and 4-HNE in different groups determined by semiquantitative evaluation $(n=5$ in each group). ${ }^{*} \mathrm{P}<0.05$ vs. normal control group; ${ }^{\&} \mathrm{P}<0.05$ vs. normal control+PA group; ${ }^{+} \mathrm{P}<0.01$ vs. H-FABP overexpression; ${ }^{\#} \mathrm{P}<0.01$ vs. H-FABP overexpression+PA group. Groups: Nor, normal control group; Nor+PA, normal control+PA group; si-Neg, negative siRNA control group; si-FABP, H-FABP siRNA group; si-Neg+PA, negative siRNA control+PA group; FABP+PA, H-FABP siRNA+PA group; Neg, negative control group; FABP, $\mathrm{H}-\mathrm{FABP}$ overexpression group; Neg+PA, negative control+PA group; FABP+PA, H-FABP overexpression+PA group. H-FABP, heart-type fatty acid binding protein; siRNA, small interfering RNA; 8-OHdG, 8-hydroxy-2'deoxyguanosine; 4-HNE, 4-hydroxynonenal.

Furthermore, A-FABP and E-FABP were indicated to be potential novel serum biomarkers for evaluating the progression of nephropathy and the associated cardiovascular risk in patients with type 2 diabetes (17). A previous study by our group detected H-FABP in podocytes, revealing that it was significantly higher expressed in podocytes from ORG patients than in those from normal controls (7). However, the function of H-FABP in podocytes has largely remained to be 
elucidated. The previous study by our group only identified that low expression of H-FABP protected the structure of podocytes in a PA environment (unpublished). Based on these results, the present study assessed the effect of H-FABP on lipid metabolism, inflammatory reaction and oxidative stress in podocytes.

PPAR $\alpha$ is of interest, as it has the ability to reflect the glucose-lowering and lipid-modifying properties of H-FABP knockdown (18). Substantial evidence has demonstrated that PPAR- $\alpha$ agonists have direct effects on several critical pathways contributing to the development and progression of diabetic kidney disease, and in diabetic PPAR- $\alpha$-knockout mice, diabetic nephropathy is more severe than in wild-type mice, as reviewed by Thomas et al (19). PPAR- $\alpha$ agonists reduce tubular injury, possibly by reducing interstitial macrophages in the kidney, leading to suppression of nuclear factor $\kappa \mathrm{B}$ and transforming growth factor- $\beta 1 / \mathrm{Smad} 3$ signaling (20). Mori et al (21) reported that PPAR- $\alpha$ expression was downregulated in murine doxorubicin-induced nephropathy and a PPAR- $\alpha$ agonist partially ameliorated the disorder likely induced through stabilization of nephrin expression and suppression of apoptosis in podocytes. PPAR- $\alpha$ has renoprotective effects in drug-associated kidney injury. PPAR- $\alpha$ deficiency exacerbates doxorubicin-associated renal injury, in part through increasing podocyte apoptosis (22).

ACOX3 is a key enzyme in the regulation of lipid homeostasis and may inhibit its target gene PPAR $\alpha$ (23). The results of the present study indicated that overexpression of H-FABP aggravated lipid metabolism derangement. Overexpression of H-FABP increased ACOX3 and reduced the expression of PPAR- $\alpha$ in podocytes, so that podocyte lesions were induced by the external factor PA. Inhibiting H-FABP expression protected podocytes from PA-induced injury by reducing ACOX 3 and increasing PPAR- $\alpha$ expression.

As an inflammatory index, MCP-1 increases cellular motility and causes actin cytoskeleton rearrangement (24). MCP-1 is also known to stimulate increased podocytes motility and cause a podocyte monolayer to become more permeable to albumin (25), which may have a role in the pathogenesis of albuminuria in vivo. When type 1 diabetes was induced in MCP-1 knockout mice by streptozotocin over up to 18 weeks, they were largely protected from developing nephropathy manifesting as albuminuria, elevated plasma creatinine, hypertrophy, tubular injury and renal fibrosis (26). The results of the present study indicated that overexpression of H-FABP enhanced the inflammatory reaction in podocytes. Overexpression of H-FABP increased MCP-1 expression in podocytes, particularly in the presence of PA, while knockdown of H-FABP protected podocytes from PA-induced injury by reducing MCP-1 expression.

Reactive oxygen species (ROS) have critical roles in the development and progression of kidney damage (27). ORG and diabetic nephropathy are associated with the increase of proinflammatory cytokines due to increased ROS production (26). Renal lipid accumulation exhibits slowly developing chronic kidney disease and is associated with increased oxidative stress (27). ROS attack guanine bases in the DNA easily and form $8-\mathrm{OHdG}$, which binds to thymidine rather than cytosine, based on which the levels of 8-OHdG is generally regarded as a biomarker of mutagenesis consequent to oxidative stress, and as a risk factor for numerous diseases including diabetes (28). 4-HNE is also a ROS damage marker (29). In the present study, overexpression of H-FABP increased 8-OHdG and 4-HNE expression in podocytes, particularly in the presence of PA, indicating that overexpression of H-FABP aggravated ROS-induced podocyte damage. Inhibition of H-FABP expression protected podocytes from PA-induced damage by reducing oxidative stress.

In conclusion, the present study provided strong evidence that the overexpression of H-FABP in podocytes aggravated lipid metabolism derangement, inflammation and oxidative stress podocyte. However, inhibition of H-FABP expression counteracted this damage in podocyte. Although the present observations require additional investigation in animals and clinical samples, the present study highlighted the potential application of H-FABP inhibition for reducing glomerular injury, particularly lipid metabolism derangement and associated podocyte injury linked with glomerular disease, including diabetic nephropathy and obesity-associated glomerulopathy.

\section{Acknowledgements}

The present study was supported by the Fujian Provincial Nature and Science Foundation (grant no. 2016J01630), the Fujian Provincial Health and Family Planning Commission Young and Middle Aged Backbone Personnel Training Project (grant no. 2016-ZQN-84) and the Xiamen Science and Technology Project (grant no. 3502Z20174078).

\section{References}

1. Sieber J and Jehle AW: Free Fatty acids and their metabolism affect function and survival of podocytes. Front Endocrinol (Lausanne) 5: 186, 2014.

2. Kampe K, Sieber J, Orellana JM, Mundel P and Jehle AW: Sesceptbility of podocytes to palmitic acid is regulated by fatty acid oxidation and inversely depends on acetyl-coA carboxylases 1and 2. Am J Physiol Renal Physiol 306: 401-409, 2014.

3. Murea M, Freedman BI, Parks JS, Antinozzi PA, Elbein SC and Ma L: Lipotoxicity in diabetic nephropathy: The potential role of fatty acid oxidation. Clin J Am Soc Nephrol 5: 2373-2379, 2010.

4. Kim EY, Roshanravan H and Dryer SE: Corrigendum to 'Syndecan-4 ectocdomain evokes mobilization of podocyte TRPC6 channels and their associated pathways: An essential role for integrin signaling'. Biochim Biophys Acta 1853: 3294, 2015.

5. Yamamoto T, Yamamoto A, Watanabe M, Matsuo T, Yamazaki N Kataoka M, Terada H and Shinohara Y: Classification of FABP isoforms and tissues based on quantitative evaluation of transcript levels of these isoforms in various rat tissues. Biotechnol Lett 31: 1695-1701, 2009.

6. Kimura H, Fujii H, Suzuki S, Ono T, Arakawa M and Gejyo F: Lipid-binding proteins in rat and human kidney. Kidney Int 56: S159-162, 1999.

7. Huimei Chen, Chunxia Zheng, Qing Gao, Yongchun Ge and Zhihong Liu: Heart-type fatty acid binding protein is associated with proteinuria in obesity. PLoS One 7: e45691, 2012.

8. Mundel P, Reiser J, Zúñiga Mejía Borja A, Pavenstädt H, Davidson GR, Kriz W and Zeller R: Rearrangements of the cytoskeleton and cell contacts induce processformation during differentiation of conditionally immortalized mouse podocyte cell lines. Exp Cell Res 236: 248-258, 1997.

9. Livak KJ and Schmittgen TD: Analysis of relative gene expression data using real-time quantitative PCR and the 2(-Delta Delta C(T)) method. Methods 25: 402-408, 2001.

10. Ockner RK and Manning JA: Fatty acid-binding protein in small intestine. Identification, isolation, and evidence for its role in cellular fatty acid transport. J Clin Invest 54: 326-338, 1974. 
11. Mishkin S, Stein L, Gatmaitan Z and Arias IM: The binding of fatty acids to cytoplasmic proteins: Binding to $\mathrm{Z}$ protein in liver and other tissues of the rat. Biochem Biophys Res Commun 47: 997-1003, 1972.

12. Matsumata $\mathrm{M}$, Inada $\mathrm{H}$ and Osumi $\mathrm{N}$ : Fatty acid binding proteins and the nervous system: Their impact on mentalconditions. Neurosci Res 102: 47-55, 2016.

13. Storch J and Thumser AE: Tissue-specific functions in the fatty acid-binding protein family. J Biol Chem 285: 32679-32683, 2010

14. Hertzel AV and Bernlohr DA: The mammalian fatty acid-binding protein multigene family: Molecular and genetic insights into function. Trends Endocrinol Metab 11: 175-180, 2000.

15. Kamijo-Ikemori A, Sugaya T, Matsui K, Yokoyama T and Kimura K: Roles of human liver type fatty acid binding protein in kidney disease clarified using hL-FABP chromosomal transgenic mice. Nephrology (Carlton) 16: 539-544, 2011.

16. Guan Y, Zhang Y, Schneider A, Davis L, Breyer RM and Breyer MD: Peroxisome proliferator-activated receptor-gamma activity is associated with renal microvasculature. Am J Physiol Renal Physiol 281: F1036-F1046, 2001.

17. Yeung DC, $\mathrm{Xu} \mathrm{A}$, Tso AW, Chow WS, Wat NM, Fong $\mathrm{CH}$ Tam S, Sham PC and Lam KS: Circulating levels of adipocyte and epidermal fatty acid-binding proteins in relation to nephropathy staging and macrovascular complications in type 2 diabetic patients. Diabetes Care 32: 132-134, 2009.

18. Bénardeau A, Verry P, Atzpodien EA, Funk JM, Meyer M, Mizrahi J, Winter M, Wright MB, Uhles S and Sebokova E: Effects of the dual PPAR- $\alpha / \gamma$ agonist aleglitazar on glycaemic control and organ protection in the Zucker diabetic fatty rat. Diabetes Obes Metab 15: 164-174, 2013.

19. Thomas MC, Jandeleit-Dahm KA and Tikellis C: The renoprotective actions of peroxisome proliferator-activated receptors agonists in diabetes. PPAR Res 2012: 456529, 2012.

20. Li L, Emmett N, Mann D and Zhao X: Fenofibrate attenuates tubulointerstitial fibrosis and inflammation through suppression of nuclear factor-kappaB and transforming growth factor-beta1/Smad3 in diabetic nephropathy. Exp Biol Med (Maywood) 235: 383-391, 2010.
21. Mori K, Mukoyama M and Nakao K: PPAR- $\alpha$ transcriptional activity is required to combat doxorubicin-induced podocyte injury in mice. Kidney Int 79: 1274-1276, 2011.

22. Zhou Y, Kong X, Zhao P, Yang H, Chen L, Miao J, Zhang X, Yang J, Ding J and Guan Y: Peroxisome proliferator-activated receptor- $\alpha$ is renoprotective in doxorubicin-induced glomerular injury. Kidney Int 79: 1302-1311, 2011.

23. Madureira TV, Castro LF and Rocha E: Acyl-coenzyme A oxidases 1 and 3 in brown trout (Salmo trutta f. fario): Can peroxisomal fatty acid $\beta$-oxidation be regulated by estrogen signaling? Fish Physiol Biochem 42: 389-401, 2016.

24. Zoja C, Corna D, Locatelli M, Rottoli D, Pezzotta A, Morigi M, Zanchi C, Buelli S, Guglielmotti A, Perico N, et al: Effects of MCP-1 inhibition by bindarit therapy in a rat model of polycystic kidney disease. Nephron 129: 52-61, 2015.

25. Chung CH, Fan J, Lee EY, Kang JS, Lee SJ, Pyagay PE, Khoury CC, Yeo TK, Khayat MF, Wang A and Chen S: Effects of tumor necrosis factor- $\alpha$ on podocyte expression of monocyte chemoattractant protein-1 and in diabetic nephropathy. Nephron Extra 5: 1-18, 2015.

26. Awad AS, Kinsey GR, Khutsishvili K, Gao T, Bolton WK and Okusa MD: Monocyte/macrophage chemokine receptor CCR2 mediates diabetic renal injury. Am J Physiol Renal Physiol 301: F1358-F1366, 2011.

27. Park S, Kim CS, Lee J, Suk Kim J and Kim J: Effect of regular exercise on the histochemical changes of d-galactose-induced oxidative renal Injury in high-fat diet-fed rats. Acta Histochem Cytochem 46: 111-119, 2013.

28. Nasif WA, Mukhtar MH, Nour Eldein MM and Ashgar SS Oxidative DNA damage and oxidized low density lipoprotein in Type II diabetes mellitus among patients with Helicobacter pylori infection. Diabetol Metab Syndr 8: 34 , 2016.

29. Bin P, Shen M, Li H, Sun X, Niu Y, Meng T, Yu T, Zhang X, Dai Y, Gao W, et al: Increased levels of urinary biomarkers of lipid peroxidation products among workers occupationally exposed to diesel engine exhaust. Free Radic Res 50: 820-830, 2016. 